\title{
Aleitamento materno até o sexto mês de vida em municípios da Rede Mãe Paranaense
}

\author{
Breastfeeding until the sixth month of life in municipalities in the Parana Mothers Network
}

Lactancia materna hasta el sexto mes de vida en los municipios de la Red Madre de Paraná

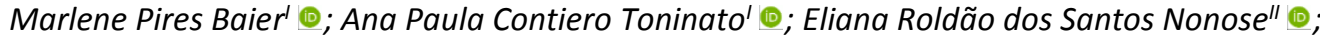 \\ Adriana Zilly' ๑; Helder Ferreiral ${ }^{\circledR}$; Rosane Meire Munhak da Silva' @
}

\begin{abstract}
'Universidade Estadual do Oeste do Paraná, Foz do Iguaçu, PR, Brasil; "Hospital Estadual do Oeste do Paraná, Foz do lguaçu, PR, Brasil
\section{RESUMO}

Objetivo: avaliar a prevalência do aleitamento materno em municípios da Rede Mãe Paranaense e identificar fatores relacionados a sua prática até o sexto mês de vida da criança. Método: estudo exploratório, prospectivo de abordagem quantitativa, com 280 lactantes, realizada entre julho de 2017 e junho de 2018, com aplicação de questionários na maternidade, 24 horas pós-parto e durante visita domiciliar aos 6 meses de vida da criança. Resultados: o aleitamento materno no sexto mês foi $7,9 \%$ exclusivo, $38,2 \%$ predominante e $30,7 \%$ misto. As consultas de puericultura estiveram associadas com o aleitamento materno, e o retorno ao trabalho foi apontado como a principal dificuldade para continuidade da amamentação. Conclusão: a prevalência do aleitamento materno exclusivo está aquém do preconizado, sendo fundamental o planejamento de ações de promoção e proteção à amamentação por meio de uma rede de apoio social, familiar e da equipe multiprofissional.

Descritores: Aleitamento Materno; Saúde da Criança; Saúde Pública; Enfermagem.
\end{abstract}

\section{ABSTRACT}

Objective: to evaluate the prevalence of breastfeeding in municipalities of the Paraná Mothers Network and to identify factors related to the practice until the child's sixth month of life. Method: this quantitative, exploratory, prospective study of 280 lactating mothers was conducted between July 2017 and June 2018 using questionnaires applied at the maternity hospital 24 hours postpartum and during home visits at six months of the child's life. Results: in the sixth month, $7.9 \%$ used breastfeeding exclusively, 38.2\%, predominantly and $30.7 \%$, a mix. Childcare appointments were associated with breastfeeding, while returning to work was identified as the main difficulty in continuing breastfeeding. Conclusion: the prevalence of exclusive breastfeeding was lower than recommended, and it is essential to plan actions to promote and protect breastfeeding through social and family support networks, and the multidisciplinary team.

Descriptors: Breast Feeding; Child Health; Public Health; Nursing.

\section{RESUMEN}

Objetivo: evaluar la prevalencia de la lactancia materna en los municipios de la Red de Madres de Paraná e identificar factores relacionados con la práctica hasta el sexto mes de vida del niño. Método: este estudio cuantitativo, exploratorio y prospectivo de 280 madres lactantes se realizó entre julio de 2017 y junio de 2018 mediante cuestionarios aplicados en la maternidad 24 horas posparto y durante las visitas domiciliarias a los seis meses de vida del niño. Resultados: en el sexto mes el 7,9\% utilizó lactancia materna exclusiva, el 38,2\% predominantemente y el 30,7\% mixta. Las citas de cuidado infantil se asociaron con la lactancia materna, mientras que el regreso al trabajo se identificó como la principal dificultad para continuar con la lactancia. Conclusión: la prevalencia de la lactancia materna exclusiva fue menor a la recomendada, y es fundamental planificar acciones para promover y proteger la lactancia materna a través de las redes de apoyo social, familiar y el equipo multidisciplinario. Descriptores: Lactancia Materna; Salud de los Niños; Salud Pública; Enfermería.

\section{INTRODUÇÃO}

O leite materno é reconhecido como a melhor fonte de alimentação da criança, com os nutrientes necessários para o crescimento e desenvolvimento infantil, além de proteger contra infecções, redução da ocorrência de diabetes tipo 2 e obesidade, podendo ainda influenciar na inteligência. Para as lactantes, oferece proteção contra o câncer de mama, ovário e diabetes tipo $2^{1}$.

A amamentação em níveis ideais, tem potencial para prevenir 823 mil mortes anualmente de crianças menores de cinco anos e 20.000 mortes por câncer de mama ${ }^{1}$. Considerando isto, a Organização Mundial de Saúde (OMS) e o Ministério da Saúde (MS) recomendam o Aleitamento Materno Exclusivo (AME), sem acréscimo de líquidos ou alimentos até o sexto mês de vida da criança, podendo ser continuado até os dois anos ou mais, junto com a alimentação complementar ${ }^{2,3}$.

Dados preliminares do Estudo Nacional de Alimentação e Nutrição Infantil (ENANI-2019) mostram que aumentou o número de crianças amamentadas exclusivamente até seis meses no Brasil, a prevalência de AME foi de $45,7 \%$, com maior frequência no Sul $(53,1 \%)$ e menor no Nordeste $(38,0 \%)^{4}$.

Agradecimento ao Conselho Nacional de Desenvolvimento Científico e Tecnológico - CNPq. Processo no 407508/2016-3.

Autora correspondente: Eliana Roldão dos Santos Nonose. E-mail: eliananonose@hotmail.com

Editora responsável: Juliana Amaral Prata 
A prática do Aleitamento Materno (AM) pode ser determinada por fatores individuais relativos aos recémnascidos, mães e famílias, até determinantes contextuais como a realidade socioeconômica, capacitação dos profissionais de saúde, serviços e políticas públicas, sendo um processo multifatorial, impregnado de ideologias e valores culturais ${ }^{5}$.

Conhecer os fatores que podem contribuir para o sucesso do aleitamento é relevante. Revisão sistemática de estudos epidemiológicos conduzidos no Brasil, encontrou 36 fatores associados ao AME, com destaque a melhores condições sociodemográficas, ausência de trabalho materno e não utilização de chupetas ${ }^{6}$.

Neste contexto, a promoção, proteção e apoio ao AM são ações que devem ser contempladas no eixo estratégico da Política Nacional de Atenção Integral à Saúde da Criança (PNAISC) para reforçar e incentivar o AME ${ }^{7}$. Congruente aos esforços para promoção do AME e redução da mortalidade infantil, o Brasil é signatário aos Objetivos do Desenvolvimento Sustentável ${ }^{8}$.

No Paraná, com vistas a reduzir a morbimortalidade materna e infantil, foi implantada a Rede Mãe Paranaense (RMP) em 2012, que propõe a organização da atenção materno-infantil, com apoio e promoção do $\mathrm{AM}^{9,10}$. Estudos anteriores a implantação da Rede, identificaram a prevalência de AME entre 30,0\% e 46,1\% em menores de seis meses ${ }^{11,12}$

Deste modo, conhecer a prevalência do AM após a implantação da RMP é importante, considerando a necessidade de avaliar as ações e contribuições para a promoção e proteção da amamentação, em busca de melhorar os indicadores de saúde materno-infantil.

Em reposta aos questionamentos: "Qual a prevalência e como ocorre a prática do AM em menores de seis meses de vida? Quais fatores contribuem ou dificultam a amamentação após a RMP?", o objetivo deste estudo foi avaliar a prevalência do AM em municípios da RMP e identificar fatores relacionados a sua prática até o sexto mês de vida da criança.

\section{MÉTOdo}

Estudo exploratório, prospectivo, quantitativo, desenvolvido na nona Regional de Saúde do Paraná, inserida na RMP. Essa regional de saúde é composta por nove municípios, situados na faixa de fronteira com o Paraguai e Argentina, com população estimada de 390.220 habitantes $^{13}$.

Para o cálculo amostral, considerou-se o número de partos ocorridos em 2016 nos municípios do estudo, um total de 6.317 nascimentos, sendo então considerado $\mathrm{N}$ tamanho (número de elementos) da população; $\mathrm{n}$ tamanho (número de elementos) da amostra; no uma primeira aproximação para o tamanho da amostra; E0 erro amostral tolerável, utilizando as seguintes fórmulas ${ }^{14}$ :

- $\mathrm{n} 0=1 /(\mathrm{EO}) 2 \cdot 0,05=400$

- $\mathrm{n}=\mathrm{N} \cdot \mathrm{n} 0 / \mathrm{N}+\mathrm{nO}$

Considerou-se uma margem de erro de 5\%, e nível de confiança de 95\%. Definiu-se $10 \%$ como margem de segurança, tendo em vista que poderiam ocorrer perdas na coleta de dados.

A pesquisa foi realizada em duas etapas, na primeira foram participantes 397 puérperas e na segunda 280, uma vez que não foi possível localizar todas as participantes para realização da visita domiciliar, devido a mudança de telefone, endereço e por insucesso de contato após três tentativas.

Foram incluídas as puérperas que realizaram o parto nas maternidades durante o período do estudo, independentemente da idade materna; sem agravos e/ou problemas no parto ou puerpério que pudesse impedir sua participação. Puérperas com bebês prematuros também foram incluídas, assim, houve participantes com crianças que nasceram com 33 semanas, sendo esta a menor idade gestacional, visto que crianças com idade gestacional inferior foram encaminhadas aos cuidados intensivos e ou intermediários.

Foram excluídas puérperas com agravos e/ou problemas de saúde mental descritos no prontuário; residentes em outros países, por considerar as dificuldades de realização da visita domiciliar em outro país; e puérperas com seus recém-nascidos hospitalizados em cuidados intensivos.

Os dados foram coletados por acadêmicos de enfermagem e pós-graduandos em saúde pública, treinados previamente por pesquisadores enfermeiros, com experiência em saúde materno-infantil.

$\mathrm{Na}$ primeira etapa, realizou-se a busca pelas participantes em três instituições hospitalares, situadas em municípios distintos, os quais são referência para o atendimento de gestação de risco habitual, intermediário e alto. Ocorreu no setor de alojamento conjunto, no período pós-parto, entre 24 e 48 horas após o nascimento da criança, com objetivo de estabelecer um primeiro contato com a puérpera e verificar sua disponibilidade para a visita domiciliar aos seis meses de vida do bebê. 
Apresentou-se os objetivos da pesquisa, e após assinatura no Termo de Consentimento Livre e Esclarecido (TCLE), aplicou-se um questionário estruturado que foi construído e validado previamente por especialistas, com variáveis como: i) sociodemográficas: endereço/telefone; idade; renda familiar; etnia; escolaridade materna; situação conjugal, ii) variáveis obstétricas/neonatais: número de gestações anteriores; número de consultas de pré-natal; tipo de parto; idade gestacional; peso ao nascer. As informações foram confirmadas por meio da verificação de prontuários e do cartão de saúde da gestante.

A segunda etapa ocorreu aproximadamente seis meses após o nascimento da criança, por meio de uma visita domiciliar, agendada por via telefônica. Dois membros da equipe de pesquisa, que se dirigiam até a residência da participante, para aplicação da segunda parte do questionário, com variáveis: duração do AME; tipo de aleitamento praticado até o sexto mês; idade de introdução de alimentos/chás e/ou água; visita domiciliar pela equipe de saúde; avaliação da mamada por profissionais de saúde; orientações recebidas sobre AM. Embora houvesse a presença de outros familiares no domicílio, estes não participaram.

Ressalta-se que foi considerado o período de análise de seis meses, tendo em vista a recomendação da OMS para manutenção do AME até esse período, para esta pesquisa, este período foi considerado aproximado.

O tipo de aleitamento fornecido à criança até o sexto mês foi classificado em AME, quando somente ofertado leite materno; Aleitamento Materno Predominante, além do leite materno, também é oferecido a criança, chás, sucos, água; Aleitamento Misto, quando a criança recebe leite materno e outros tipos de leite; e Leite Artificial, quando ocorre a oferta deste ${ }^{3}$.

As variáveis selecionadas e coletadas foram digitadas e classificadas conforme os indicadores da literatura, sendo relacionadas a contribuição dos fatores sociodemográficos, obstétricos e de seguimento da atenção básica.

Para analisar os dados, foi realizada análise descritiva e utilizado o Teste de Qui-quadrado, complementado pela análise dos resíduos ajustados, com nível significância de $5 \%$ para verificar a associação entre a variável dependente (tipo de aleitamento), com as demais variáveis independentes, utilizando-se o programa XLStat2014 ${ }^{\circledR}$.

Este estudo é parte de um projeto maior intitulado "Rede mãe paranaense na perspectiva da usuária: o cuidado da mulher no pré-natal, parto, puerpério e da criança" o qual foi submetido e aprovado pelo Comitê de Ética em Pesquisa da Universidade Estadual de Londrina, sob o parecer de aprovação no 2.053.304. Cabe destacar que, para puérperas com idade inferior a 18 anos, um responsável acompanhante maior de idade autorizou sua participação e assinou o TCLE.

\section{RESULTADOS}

Participaram 280 lactantes, com as variáveis sociodemográficas e obstétricas/neonatais, de acordo com o tipo de alimentação da criança, apresentadas na Tabela 1.

Verifica-se 197 participantes (70,3\%) na faixa etária entre 20 e 24 anos, 152 (54,3\%) de etnia branca e 195 (69,6\%) com escolaridade acima de oito anos de estudo. Quase a totalidade, 257 (91,8\%) tem companheiro, 126 (45\%) com renda familiar de um a dois salários mínimos e pouco mais da metade, 147 (52,5\%) não exerce atividade remunerada.

A prevalência do AME ao sexto mês de vida da criança foi de 22 (7,9\%), 107 (38,2\%) para o aleitamento materno predominante, enquanto $86(30,7 \%)$ encontravam-se em aleitamento misto e $65(23,2 \%)$ recebiam apenas leite artificial.

Embora não tenham sido identificadas associações com significância estatística, nota-se que a maior proporção de crianças em AM, sem outros tipos de leite, até o sexto mês, foram de mães menores de 19 anos, de etnia branca, com escolaridade de até oito anos, com companheiro, renda mensal entre um e dois salários mínimos, e maioria sem exercer trabalho remunerado. Não houve associações estatísticas para as variáveis obstétricas/neonatais, mas a maior prevalência do aleitamento ocorreu para primíparas, submetidas ao parto vaginal, com no mínimo três consultas de pré-natal, bebês nascidos a termo ( $37-41$ semanas) ou pós-termo ( $\geq 42$ semanas), e com baixo peso ( $\leq 2499 \mathrm{~g}$ ) ou adequado (2500-3999g).

Na Tabela 2, são apresentadas as variáveis de cuidado e segmento à saúde das mulheres e crianças, de acordo com o tipo de aleitamento ofertado à criança no sexto mês de vida.

Quase a metade das mulheres relataram dificuldades para amamentar, o que culminou no desmame, sendo significativamente menor a prevalência do AME e predominante até o sexto mês para essas mulheres, quando comparado àquelas que não relataram problemas para manter a amamentação. Para as demais variáveis de cuidado e seguimento à saúde, houve significância estatística para as consultas de puericultura, onde quem realizou oito ou mais consultas, teve maior prevalência de aleitamento materno predominante. 
TABELA 1: Variáveis sociodemográficas, neonatais e obstétricas das mulheres de acordo com o tipo de aleitamento ofertado à criança no sexto mês de vida $(n=280)$. Foz do Iguaçu, PR, Brasil, 2018.

\begin{tabular}{|c|c|c|c|c|c|c|c|c|}
\hline \multirow[b]{2}{*}{ Variáveis } & & \multicolumn{2}{|c|}{$\begin{array}{l}\text { *AME e } \\
\text { Predominante } \\
(n=129)\end{array}$} & \multicolumn{2}{|c|}{$\begin{array}{c}\text { Aleitamento } \\
\text { Misto } \\
(n=86)\end{array}$} & \multicolumn{2}{|c|}{$\begin{array}{c}\text { Leite } \\
\text { Artificial } \\
(n=65)\end{array}$} & \multirow[t]{2}{*}{$* *$ p valor } \\
\hline & & $\mathrm{n}$ & $\%$ & $n$ & $\%$ & $\mathbf{n}$ & $\%$ & \\
\hline \multirow[t]{3}{*}{ Idade } & $\leq 19$ anos & 25 & 55,6 & 8 & 17,8 & 12 & 26,7 & \\
\hline & 20 a 34 anos & 84 & 42,6 & 68 & 34,5 & 45 & 22,8 & 0,2247 \\
\hline & $\geq 35$ anos & 20 & 52,6 & 10 & 26,3 & 8 & 21,1 & \\
\hline \multirow[t]{3}{*}{ Etnia } & Branca & 68 & 52,7 & 48 & 55,8 & 36 & 55,4 & \\
\hline & Parda/ Negra & 60 & 48,0 & 38 & 30,4 & 27 & 21,6 & 0,4790 \\
\hline & Outra/Sem registro & 1,00 & 33,3 & 0 & 0,0 & 2 & 66,7 & \\
\hline \multirow[t]{2}{*}{ Escolaridade } & $\leq 8$ anos & 43 & 50,6 & 23 & 27,1 & 19 & 22,4 & 0,5739 \\
\hline & 9 ou mais & 86 & 44,1 & 63 & 32,3 & 46 & 23,6 & \\
\hline \multirow[t]{2}{*}{ Situação conjugal } & Com companheiro & 120 & 46,7 & 80 & 31,1 & 57 & 22,2 & 0,4331 \\
\hline & Sem companheiro & 9 & 39,1 & 6 & 26,1 & 8 & 34,8 & \\
\hline \multirow[t]{2}{*}{ Ocupação } & Remunerada & 52 & 39,1 & 45 & 33,8 & 36 & 27,1 & 0,0790 \\
\hline & Não remunerada & 77 & 52,4 & 41 & 27,9 & 29 & 19,7 & \\
\hline \multirow[t]{5}{*}{ Renda familiar } & $<1$ salário & 23 & 44,2 & 14 & 26,9 & 15 & 28,8 & \\
\hline & 1 a 2 salários & 63 & 50,0 & 38 & 30,2 & 25 & 19,8 & 0,9326 \\
\hline & 2 a 3 salários & 29 & 43,9 & 22 & 33,3 & 15 & 22,7 & \\
\hline & $\geq 4$ salários & 11 & 37,9 & 10 & 34,5 & 8 & 27,6 & \\
\hline & Não informou & 3 & 42,9 & 2 & 28,6 & 2 & 28,6 & \\
\hline \multirow[t]{2}{*}{ Tipo de parto } & Vaginal & 67 & 46,5 & 50 & 34,7 & 27 & 18,7 & 0,1281 \\
\hline & Cesárea & 62 & 45,6 & 36 & 26,5 & 38 & 27,9 & \\
\hline \multirow[t]{4}{*}{ Paridade } & Primeira & 52 & 49,1 & 29 & 27,4 & 25 & 23,6 & \\
\hline & Segunda & 38 & 43,7 & 31 & 35,6 & 18 & 20,7 & 0,5850 \\
\hline & Terceira & 22 & 44,9 & 13 & 26,5 & 14 & 28,6 & \\
\hline & Quarta ou mais & 17 & 44,7 & 13 & 34,2 & 8 & 21,1 & \\
\hline \multirow{3}{*}{$\begin{array}{l}\text { Consultas de pré- } \\
\text { natal }\end{array}$} & 0 a 2 & 0 & 0,0 & 5 & 71,4 & 2 & 28,6 & \\
\hline & 3 a 5 & 21 & 55,3 & 9 & 23,7 & 8 & 21,1 & 0,1371 \\
\hline & 6 ou mais & 108 & 45,9 & 72 & 30,9 & 55 & 23,2 & \\
\hline \multirow[t]{3}{*}{ Idade gestacional } & $\leq 36$ semanas & 4 & 21,05 & 9 & 47,4 & 6 & 31,6 & \\
\hline & 37 a 41 semanas & 122 & 47,64 & 76 & 29,9 & 57 & 22,4 & 0,3243 \\
\hline & $\geq 42$ semanas & 3 & 50,00 & 1 & 16,7 & 2 & 33,3 & \\
\hline \multirow[t]{3}{*}{ Peso ao nascer } & $\leq 2499 \mathrm{~g}$ & 10 & 58,8 & 1 & 5,9 & 6 & 35,3 & \\
\hline & 2500 a 3999 & 110 & 46,2 & 78 & 32,6 & 50 & 21,2 & 0,1881 \\
\hline & $\geq 4000 \mathrm{~g}$ & 9 & 36,0 & 7 & 28,0 & 9 & 36,0 & \\
\hline
\end{tabular}

*Aleitamento Materno Exclusivo, ${ }^{* *}$ Teste qui-quadrado $\mathrm{p}$ valor $<0,05$ 
TABELA 2: Variáveis de cuidado e segmento à saúde das mulheres e crianças, de acordo com o tipo de aleitamento ofertado à criança no sexto mês de vida $(n=280)$. Foz do Iguaçu, PR, Brasil, 2018.

\begin{tabular}{|c|c|c|c|c|c|c|c|c|}
\hline \multirow[t]{2}{*}{ Variáveis } & & \multicolumn{2}{|c|}{$\begin{array}{l}\text { *AME e Predominante } \\
\qquad(\mathrm{n}=129)\end{array}$} & \multicolumn{2}{|c|}{$\begin{array}{l}\text { Alimentação } \\
\text { Mista }(n=86)\end{array}$} & \multicolumn{2}{|c|}{$\begin{array}{l}\text { Leite Artificial } \\
\qquad(n=65)\end{array}$} & \multirow[t]{2}{*}{$* *$ p valor } \\
\hline & & $n$ & $\%$ & $\mathrm{n}$ & $\%$ & $n$ & $\%$ & \\
\hline \multirow[t]{2}{*}{ Visita domiciliar } & Sim & 35 & 39,3 & 31 & 34,8 & 23 & 25,8 & \multirow{2}{*}{0,3017} \\
\hline & Não & 94 & 49,2 & 55 & 28,8 & 42 & 22 & \\
\hline \multirow{2}{*}{$\begin{array}{l}\text { Avaliação mamadas } \\
(n=268)\end{array}$} & Sim & 63 & 50 & 38 & 30,2 & 25 & 19,8 & \multirow{2}{*}{0,477} \\
\hline & Não & 62 & 43,7 & 44 & 31 & 36 & 25,3 & \\
\hline \multirow{2}{*}{$\begin{array}{l}\text { Dificuldades que } \\
\text { levaram ao desmame }\end{array}$} & Sim & 13 & 12,7 & 32 & 31,4 & 57 & 55,9 & \multirow{2}{*}{0,0001} \\
\hline & Não & 116 & 65,2 & 54 & 30,3 & 8 & 4,5 & \\
\hline \multirow{2}{*}{$\begin{array}{l}\text { Ajuda para superar } \\
\text { dificuldades }(n=102)\end{array}$} & Sim & 2 & 7,4 & 6 & 22,2 & 19 & 70,4 & \multirow{2}{*}{0,2039} \\
\hline & Não & 11 & 14,7 & 26 & 34,7 & 38 & 50,7 & \\
\hline \multirow{5}{*}{$\begin{array}{l}\text { Orientação introdução } \\
\text { alimentar }(n=264)\end{array}$} & Sim & 100 & 49,7 & 56 & 27,9 & 45 & 22,4 & \multirow{5}{*}{0,1331} \\
\hline & Não & 23 & 36,5 & 25 & 39,7 & 15 & 23,8 & \\
\hline & Uma & 8 & 25 & 14 & 43,7 & 10 & 31,2 & \\
\hline & Duas & 11 & 40,7 & 14 & 51,8 & 2 & 7,4 & \\
\hline & Três & 13 & 40,6 & 16 & 50 & 3 & 9,4 & \\
\hline \multirow{5}{*}{ Puericulturas } & Quatro & 9 & 27,3 & 10 & 30,3 & 14 & 42,4 & \multirow[t]{5}{*}{0,0002} \\
\hline & Cinco & 19 & 51,3 & 7 & 18,9 & 11 & 29,7 & \\
\hline & Seis & 15 & 48,4 & 11 & 35,5 & 5 & 16,1 & \\
\hline & Sete & 25 & 54,4 & 8 & 17,4 & 13 & 28,3 & \\
\hline & Oito ou mais & 15 & 75 & 2 & 10 & 3 & 15 & \\
\hline
\end{tabular}

*Aleitamento Materno Exclusivo, ${ }^{* *}$ Teste qui-quadrado

Mais da metade das mulheres já havia introduzido leite artificial até o sexto mês da criança e $45,7 \%$ ofertaram leite em pó ou líquido aos quatro meses. O profissional mais citado para introdução do leite artificial foi o pediatra $(15,3 \%)$. A redução na produção de leite e o retorno ao trabalho foram as dificuldades mais citadas que levaram ao desmame (Figura 1).

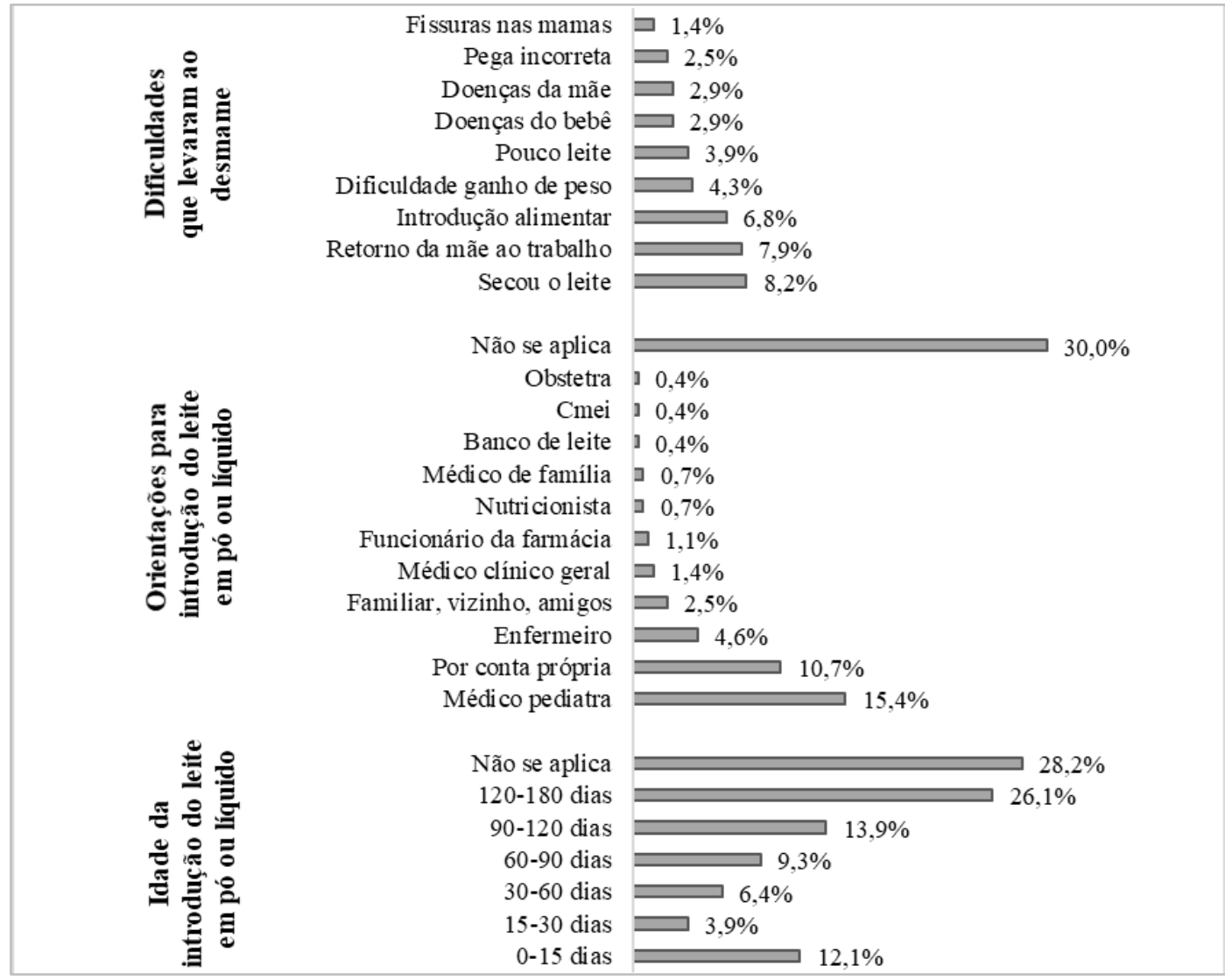

*Cmei: Centro Municipal de Educação Infantil.

Figura 1: Dificuldades que levaram ao desmame precoce e introdução do leite artificial ( $n=280)$. Foz do Iguaçu, PR, Brasil, 2018. 
Quanto à introdução de chá ou água antes dos seis meses, 59,2\% já ofertaram no quarto mês e 32,8\% de quatro a seis meses. O principal motivo relatado foi acreditar que o bebê sentia sede e pela introdução de alimentos.

\section{DISCUSSÃO}

Os resultados deste estudo mostraram que menos de $10 \%$ das crianças foram amamentadas exclusivamente até o sexto mês, sendo essa prevalência considerada insatisfatória segunda a OMS ${ }^{3}$, estando aquém de outros estudos realizados no Brasil que apontaram prevalência de AME de $20 \%$ a $36,6 \%$, conforme pesquisa nacional que avaliou a tendência do $\mathrm{AM}$ nos últimos 30 anos no país ${ }^{15,16}$

Com respeito as taxas de aleitamento predominante, somadas ao AME, cerca da metade das crianças encontravase recebendo leite materno, corroborando com os achados no restante do país, que apontavam prevalência de $50 \%{ }^{15,16}$.

Embora no Brasil tenha-se avançado para maior tempo de permanência do AM, devido a implantação de políticas governamentais, ainda não é suficiente. Uma meta-análise demonstrou que um aumento de $10 \%$ na duração do AME tem potencial para redução de custos ao sistema de saúde e da morbidade infantil, além de ganhos cognitivos para a criança na vida adulta, com potencial por salvar a vida de cerca de seis milhões de crianças por ano no mundo ${ }^{17}$.

No que se refere as variáveis sociodemográficas, não foram encontradas associações estatísticas com a prevalência da amamentação, embora alguns estudos apontem o gradiente socioeconômico com potencial para influenciar nas condições de saúde da população, podendo promover ou dificultar a amamentação de acordo com a renda, escolaridade, idade materna e etnia ${ }^{5,6,16}$

A maior proporção de crianças em AM até o sexto mês de vida, foi de mães com idade inferior a 19 anos. Porém resultados de outras pesquisas mostram que a interrupção precoce do AME e a inclusão de alimentos artificiais à dieta da criança constituem-se práticas comuns entre mães adolescentes ${ }^{17-19}$.

Em se tratando de escolaridade, mães com menos de oito anos de estudo tiveram proporção maior de aleitamento, resultado divergente de outras investigações que apontam maior amamentação entre as mães com maior escolaridade ${ }^{17,19,20}$

A renda familiar também não estava associada com a amamentação, diferente de outros estudos em que a adesão à amamentação respondeu positivamente a melhor renda familiar ${ }^{6,16,20}$. Todavia, houve maior prevalência entre aquelas que exercem ocupação não remunerada, convergindo com outros achados onde as mulheres que não trabalham fora amamentam por mais tempo ${ }^{16,20}$.

A presença de companheiro mostrou-se importante para maior prevalência do AM. Estudos apontam que o apoio do companheiro na amamentação tem relação direta com a manutenção e duração da amamentação ${ }^{6,20}$

Quanto às variáveis obstétricas, não se observou associações com o aleitamento, embora tenha ocorrido maior predominância entre as mulheres primíparas, com parto vaginal e, no mínimo, três consultas de pré-natal. A literatura aponta que uma possível explicação para a associação da paridade com o AME é o papel da experiência materna anterior com o aleitamento, pois mulheres com experiência anterior têm maior chance de realizar AME nos seis meses após o nascimento ${ }^{15,20}$. Sobre o tipo de parto, a cesariana mostrou-se associada à menor prevalência do AM por um estudo e pode ser relacionada a outros fatores de risco, como não nascer em uma maternidade com Iniciativa Hospital Amigo da Criança e não amamentar na primeira hora de vida ${ }^{21}$. Em relação as consultas de pré-natal, estas mostraram-se importantes para o seguimento do AM, assim como na pesquisa realizada em Porto Alegre que demonstrou resultados favoráveis para o AME para mulheres com mais de oito consultas ${ }^{22}$.

As características neonatais envolvidas com o AME foram baixo peso ou peso adequado, e o nascimento a termo e o pós-termo. Outras pesquisas também não encontraram associações significativas destes dados com o AM, do mesmo modo, identificaram maior prevalência de AME para o grupo de crianças nascidas a termo e com peso adequado ao nascer ${ }^{12,20}$.

Uma variável que demonstrou significância, como fator importante para a amamentação, foi o número de consultas de puericultura, sendo que mulheres que realizaram oito ou mais consultas, tiveram uma maior prevalência do AM, sem adição de outros tipos de leite.

A RMP preconiza que a criança realize no mínimo oito consultas no primeiro ano de vida, enfatizando as orientações para a manutenção do aleitamento e introdução de alimentação complementar quando indicado ${ }^{9}$. A puericultura é um momento prioritário no cuidado dispensado à criança e família, em que deve haver troca de experiências e fortalecimento de vínculo entre os envolvidos, para que as mães se sintam seguras para realizar o cuidado à criança ${ }^{7}$.

Nesse sentido, destaca-se a atuação do enfermeiro, que exerce papel importante na promoção e proteção ao AM que se inicia durante a assistência pré-natal e se estende nas consultas de puericultura, sendo essencial que este profissional conheça a prevalência do aleitamento, bem como os fatores que promovem ou dificultem a manutenção 
da amamentação, para direcionar o cuidado ao binômio mãe-filho, para favorecer a lactação e prevenir o desmame precoce. Pode ainda proporcionar à mulher o resgate de sua autonomia, transmitindo segurança e tranquilidade para que a mãe desenvolva seu potencial no nascimento e para o cuidado do filho ${ }^{23,24}$.

Quase a totalidade das crianças recebeu água ou chá antes dos seis meses de vida, reforçado pela crença de que a criança sentia sede ou pela introdução de outros alimentos precocemente. Resultado semelhante foi encontrado onde $70 \%$ das crianças até seis meses já haviam recebido algum tipo de alimento ou líquido ${ }^{16}$.

A introdução precoce de outros líquidos e alimentos pode ocorrer pela falta de informação materna, reforçados pela crença que somente o leite materno não é suficiente para o ganho de peso adequado, ou que não seja capaz de saciar a sede da criança ${ }^{16,25}$. Vale ressaltar que a introdução de alimentos antes dos seis meses, pode resultar em infeções gastrointestinais e respiratórias, além do risco de prejuízos ao crescimento e desenvolvimento, uma vez que as necessidades nutricionais do lactente podem não ser atendidas devido a não oferta do leite materno ${ }^{16}$.

Sobre à descontinuidade da amamentação, quase metade das participantes proferiu dificuldades que levaram ao desmame precoce. Este grupo de mulheres introduziu o leite artificial em maior frequência, quando comparadas as lactantes que não relataram dificuldades durante a amamentação. Corroborando com estes achados, o estudo realizado em um serviço especializado em amamentação, revelou que as dificuldades apresentadas pelas mulheres interferem na manutenção do $\mathrm{AME}^{26}$.

A redução da produção de leite e o retorno ao trabalho foram as dificuldades mais citadas pelas mulheres para a continuidade da amamentação. O trabalho materno é reconhecido como um dos principais motivos para não amamentar ou pelo desmame precoce ${ }^{17}$. Assim, faz-se necessário as políticas de proteção à amamentação como a licença maternidade, tendo em vista a crescente participação feminina no mercado de trabalho, aqui representado por quase metade das mulheres exercendo trabalho remunerado.

Uma investigação realizada no Rio de Janeiro constatou que a prevalência de AME foi 91\% maior para as mães que recebiam licença maternidade, quando comparado as que não recebiam ${ }^{27}$. Outro estudo realizado nas capitais brasileiras também apontou que a licença maternidade contribuiu para o aumento da prevalência da amamentação ${ }^{28}$.

No Brasil, muito embora tenha sido aprovada em 2008 a ampliação da licença maternidade para 180 dias, a concessão do benefício é de caráter voluntário por parte das empresas privadas, o que faz com que a adesão ainda seja baixa, onde grande parte das mães trabalhadoras acabam retornado ao trabalho após 120 dias, em torno dos quatro meses de vida da criança ${ }^{28,29}$, o que pode resultar na interrupção do aleitamento.

Um aspecto a destacar neste estudo, é que o profissional que mais orientou a introdução do aleitamento artificial foi o pediatra, provavelmente pelo retorno da mulher ao trabalho, semelhante aos achados encontrados em diversas cidades do país ${ }^{30}$.

As recomendações por meio de profissionais da saúde parecem preocupantes em relação ao desmame precoce, podendo ser gerado por falta de capacitação no manejo da amamentação ${ }^{30}$. Um estudo realizado com profissionais de saúde, quanto as práticas para a promoção do AM após a implantação da RMP, revelou que há desconhecimento sobre a adesão do aleitamento na localidade onde atuam, e que as práticas de incentivo ao aleitamento ocorrem de maneira fragmentada, não contemplando a integralidade do cuidado do binômio mãe-filho ${ }^{24}$.

Para as mães manterem a lactação após retorno ao trabalho, é importante que o profissional de saúde estimule os familiares, em especial o companheiro, quando presente, a dividir as tarefas domésticas com a nutriz e oriente a mãe trabalhadora a adotar medidas como: praticar a ordenha e congelar o leite para uso futuro; conhecer as facilidades para a retirada e armazenamento do leite no local de trabalho (privacidade, freezer, horários, conservação) e evitar uso de mamadeiras e chupetas ${ }^{3,9}$.

É importante salientar que o sucesso do AM não é apenas responsabilidade da mulher, uma vez que a promoção do aleitamento perpassa pela responsabilidade social coletiva ${ }^{17}$.

Neste estudo, embora não tenha sido encontrado a associação de fatores sociodemográficos com a baixa prevalência do AME, resultado também encontrado em outro estudo no Paraná31 ${ }^{1}$ pode-se inferir que o desmame aconteceu principalmente pelo retorno ao trabalho, e em menor proporção pela influência cultural, presente na região por fazer parte de uma faixa de fronteira.

\section{Limitações do estudo}

Este estudo possui limitação de viés de memória, uma vez que as participantes podem não se lembrar exatamente da ordem cronológica de todos os acontecimentos referentes a amamentação e suas dificuldades. Além disso, ocorreram algumas perdas, pois não foi possível contatar todas as mulheres que participaram na primeira etapa da pesquisa, para realização de visita domiciliar, por se tratar de região de fronteira e de grande mobilidade populacional. 
Entretanto, possibilitou a identificação da prevalência do AM e os fatores relacionados à sua prática até o sexto mês de vida da criança. Esse conhecimento é fundamental para direcionar as práticas assistenciais do enfermeiro para o atendimento das necessidades de saúde da mulher e da criança, bem como na adoção de estratégias facilitadoras para a promoção do aleitamento e diminuição do desmame precoce, contribuindo com a melhoria dos indicadores de saúde dessa população, atendendo as premissas da RMP.

\section{CONCLUSÃO}

O estudo demonstrou que decorridos quase uma década da implantação da RMP, a prevalência do AME até o sexto mês encontra-se abaixo do preconizado, mas o AM predominante foi satisfatório. Fatores sociodemográficos, obstétricos e neonatais não mostraram associação significativa com o AME, mas verificou-se maior proporção de crianças em AME até o sexto mês para mães menores de 19 anos, brancas, com escolaridade de até oito anos, com companheiro, renda mensal entre um e dois salários mínimos e sem exercer trabalho remunerado. Assim como, para primíparas submetidas ao parto vaginal, com no mínimo três consultas de pré-natal, bebês nascidos a termo ou póstermo e com baixo peso ou adequado.

Constatou-se que entre as maiores dificuldades para manter a amamentação, o retorno ao trabalho foi expressivo.

A consulta de puericultura foi identificada como fator protetor para a manutenção do aleitamento, demonstrando a importância do acompanhamento dos profissionais de saúde, em especial do enfermeiro, ao desempenhar ações educativas relevantes para a promoção e manutenção do AM.

Ademais, estudos como este possibilitam criar estratégias para planejar ações que visem à proteção e apoio ao AM, com fortalecimento da rede de apoio, com a participação da família e interação com a equipe interdisciplinar, promovendo um processo de aleitamento tranquilo e bem-sucedido.

\section{REFERENCES}

1. Victora CG, Bahl R, Barros AJD, França GVA, Horton S, Krasevec J, et al. Breastfeeding in the 21st century: epidemiology, mechanisms, and lifelong effect. Lancet [Internet], 2016 [cited 2020 May 05]; 387:475-90. DOI: https://doi.org/10.1016/S01406736(15)01024-7

2. World Health Organization (WHO). Global strategy for infant and young child feeding. Geneva; 2001. (Document A54/7). [cited 2020 May 05]; Available from: https://www.who.int/nutrition/publications/infantfeeding/9241562218/en/

3. Ministério da Saúde (Br). Estratégia Nacional para Promoção do Aleitamento Materno e Alimentação Complementar Saudável no Sistema Único de Saúde: Manual de implementação. [Internet]. Brasília, 2015. [cited 2019 Oct 12]. Available from: http://bvsms.saude.gov.br/bvs/publicacoes/estrategia_nacional_promocao_aleitamento_materno.pdf

4. Universidade Federal do Rio de Janeiro (UFRJ). Estudo Nacional de Alimentação e Nutrição Infantil - ENANI-2019: Resultados preliminares - Indicadores de aleitamento materno no Brasil. UFRJ: Rio de Janeiro, 2020. 9p. Available from: https://enani.nutricao.ufrj.br/index.php/relatorios/

5. Moraes de Oliveira M, Camelo JS. Gestational, perinatal, and postnatal factors that interfere with practice of exclusive breastfeeding by six months after birth. Int. Breastfeed J. [Internet], 2017 [cited 2020 May 05]; 12:42. DOI: https://doi.org/10.1186/s13006-017-0132-y

6. Boccolini CS, Carvalho ML, Oliveira MIC. Factors associated with exclusive breastfeeding in the first six months of life in Brazil: a systematic review. Rev. Saúde Públ. [Internet], 2015 [cited 2020 May 05]; 49:91. DOI: https://doi.org/10.1590/S00348910.2015049005971

7. Ministério da Saúde (Br). Secretaria de Atenção à Saúde. Departamento de Ações Programáticas e Estratégicas. Política Nacional de Atenção Integral à Saúde da Criança: orientações para implementação [Internet]. Brasília, 2018 [cited 2020 May 10]. Available from:

http://www.saude.pr.gov.br/arquivos/File/Politica_Nacional_de_Atencao_Integral_a_Saude_da_Crianca_PNAISC.pdf

8. United Nations. Transforming our world: the 2030 Agenda for Sustainable Development [Internet]. New York, 2015 [cited 2020 Mar 03]. Available from: https://nacoesunidas.org/pos2015/ods3/

9. Secretaria de Estado da Saúde do Paraná (SESA). Linha guia rede mãe paranaense [Internet]. Paraná, 2017 [cited 2018 Nov 15 ] Available from: http://www.saude.pr.gov.br/arquivos/File/ACS/linha_guia_versao_final.pdf

10. Caldeira S, Luz MS, Tacla MTGM, Machineski GG, Silva RMM, Pinto MPV et.al. Nursing care actions in the paranaense mother network program. Rev Min Enferm [Internet], 2017 [cited 2020 May 01]; 21:e-992. DOI: http://www.dx.doi.org/10.5935/14152762.20170002

11. Ministério da Saúde (Br). Secretaria de Atenção à Saúde. Departamento de Ações Programáticas e Estratégicas. II Pesquisa de Prevalência de Aleitamento Materno nas Capitais Brasileiras e Distrito Federal / Ministério da Saúde, Secretaria de Atenção à Saúde, Departamento de Ações Programáticas e Estratégicas. Brasília (DF): Editora do Ministério da Saúde, 2009. 108 p. [cited 2020 May 01]; Available from: http://bvsms.saude.gov.br/bvs/publicacoes/pesquisa_prevalencia_aleitamento_materno.pdf

12. Demitto MO, Antunes MB, Bercini LO, Rossi RM, Torres MM, Lopes TCR et al. Prevalência e fatores determinantes do aleitamento materno exclusivo. Rev. Uningá [Internet], 2017 [cited 2020 Oct 15]; 52(1):29-33. DOI: http://revista.uninga.br/index.php/uninga/article/view/1387 
13. Instituto Paranaense de Desenvolvimento Social e Econômico (IPARDES). Caderno Estatístico dos Municípios [Internet]. Foz do Iguaçu, 2020 [cited 2020 Mar 05]. Available from:

http://www.ipardes.gov.br/cadernos/MontaCadPdf1.php?Municipio=85850\&btOk=ok

14. Pito ALBS. Epidemiologia aplicada nos serviços de saúde. 1ạ ed. São Paulo: Editora Martinari; 2012

15. Boccolini CS, Boccolini PMM, Monteiro FR, Venâncio SI, Giugliani ERJ. Breastfeeding indicators trends in Brazil for three decades. Rev. Saúde Públ. [Internet], 2017 [cited 2020 May 05]; 51:108. DOI: https://doi.org/10.11606/S15188787.2017051000029

16. Flores TR, Nunes BP, Neves RG, Wendt AT, Costa CS, Wehrmeister FC et al. Maternal breastfeeding and associated factors in children under two years: the Brazilian National Health Survey, 2013. Cad. Saúde Pública [Internet], 2017 [cited 2020 May 05]; 33(11):e00068816. DOI: https://doi.org/10.1590/0102-311x00068816

17. Rollins NC, Bhandari N, Hajeebhoy N, Horton S, Lutter CK, Martines JC et al. Why invest, and what it will take to improve breastfeeding practices? Lancet [Internet], 2016 [cited 2020 May 03]; 387(10017):491-504. 2016. DOI: https://doi.org/10.1016/S0140-6736(15)01044-2

18. Maranhão TA, Gomes KRO, Nunes LB, Moura LNB. Factors related to exclusive breastfeeding among adolescent mothers. Cad. Saude Colet. [Internet], 2015 [cited 2020 May 15]; 23(2):132-9. DOI: https://doi.org/10.1590/1414-462X201500020072

19. Alves JS, Oliveira MIC, Rito RVVF. Guidance on breastfeeding in primary health care and the association with exclusive breastfeeding. Ciênc. Saúde Colet. [Internet], 2018 [cited 2020 May 18]; 23(4):1077-88. DOI: https://doi.org/10.1590/141381232018234.10752016

20. Margotti E, Margotti W. Factors related to Exclusive Breastfeeding in Babies born in a childfriendly hospital in a capital of Northern Brazil. Saúde debate [Internet], 2017 [cited 2020 May 08]; 41(114):860-71. DOI: https://doi.org/10.1590/01031104201711415

21. Freitas MG, Werneck AL, Borim BC. Exclusive breastfeeding: adhesion and difficulties. J. Nurs. UFPE online [Internet], 2018 [cited 2020 May 10]; 12(9):2301-7. DOI: https://doi.org/10.5205/1981-8963-v12i9a234910p2301-2307-2018

22. Gasparin VA, Strada JKR, Moraes BA, Betti T, Pitilin ÉB, Santo LCE. Factors associated with the maintenance of exclusive breastfeeding in the late postpartum. Rev. Gaúcha Enferm. [Internet], 2020 [cited 2020 Oct 20]; 41(esp): e20190060. DOI: https://doi.org/10.1590/1983-1447.2020.20190060

23. Furtado MCC, Mello DF, Pina JC, Vicente JB, Lima PR, Rezende VD. Nurses' actions and articulations in child care in primary health care. Texto contexto enferm. [Internet], 2018 [cited 2020 May 15]; 27(1):e930016. DOI: http://dx.doi.org/10.1590/010407072018000930016

24. Silva RMM, França AFO, Toninato APC, Ferrari, RAP, Caldeira S, Zilly A. Promotion of breastfeeding: practices of physicians and primary health care nurses. RECOM - Rev. enferm. Cent. Oeste Min. [Internet], 2019 [cited 2020 May 10]; 9:e3335. DOI: https://doi.org/10.19175/recom.v9i0.3335

25. Campos AMS, Chaoul CO, Carmona EV, Higa R, Vale IN. Exclusive breastfeeding practices reported by mothers and the introduction of additional liquids. Rev. Latino-Am. Enfermagem [Internet], 2015 [cited 2020 May 05]; 23(2):283-90. DOI: http://dx.doi.org/10.1590/0104-1169.0141.2553

26. Carreiro JÁ, Francisco AA, Abrão ACFV, Marcacine KO, Abuchaim ESV, Coca KP. Breastfeeding diffi culties: analysis of a service specialized in breastfeeding. Acta paul. enferm. [Internet], 2018 [cited 2020 Oct 10]; 31(4):430-4. DOI: https://doi.org/10.1590/1982-0194201800060

27. Rimes KA, Oliveira MIC, Boccolini CS. Maternity leave and exclusive breastfeeding. Rev. Saúde Públ. [Internet], 2019 [cited 2020 May 13]; 53:10. DOI: https://doi.org/10.11606/S1518-8787.2019053000244

28. Monteiro FR, Buccini GS, Venâncio SI, Costa THM. Influence of maternity leave on exclusive breastfeeding. J. Pediatr. [Internet], 2017 [cited 2020 May 02]; 93(5):475-81. DOI: https://doi.org/10.1016/j.jped.2016.11.016

29. Brasil. Lei $N^{\circ} 11.770$, de 9 de setembro de 2008. Cria o Programa Empresa Cidadã, destinado à concessão de licença de maternidade mediante concessão de incentivo fiscal, e altera a Lei n ${ }^{\circ}$ 8.212, de 24 de julho de 1991. Diário Oficial da União. 10 set 2008; Seção 1:1.

30. Morais MB, Cardoso AL, Lazarini T, Mosquera EMB, Mallozi MC. Habits and attitudes of mothers of infants in relation to breastfeeding and artificial feeding in 11 brazilian cities. Rev. Paul. Pediatr. [Internet], 2017 [cited 2020 May 10]; 35(1):39-45. DOI: https://doi.org/10.1590/1984-0462/;2017;35;1;00014

31. Fernandes RC, Höfelmann DA. Intention to breastfeed among pregnant women: association with work, smoking, and previous breastfeeding experience. Ciênc. Saúde Colet. [Internet], 2020 [cited 2020 Oct 23]; 25(3):1061-72. DOI:

https://doi.org/10.1590/1413-81232020253.27922017 